\title{
EVIDENCIAS DE CONOCIMIENTO ENTRE MATEMÁTIGAS Y FÍSICA SOBRE VELOCIDAD MEDIA
}

\author{
EVIDENCE OF KNOWLEDGE BETWEEN MATHEMATICS \\ AND PHYSICS ABOUT AVERAGE SPEED
}

María del Valle Bermejo-Luna

vll.bermejo@gmail.com

Gloria Sánchez-Matamoros García

Universidad de Sevilla, Sevilla, España

\author{
gsanchezmatamoros@us.es \\ Universidad de Sevilla, Sevilla, España
}

\section{RESUMEN}

Resultados de investigaciones previas han puesto de manifiesto la complejidad de la transferencia de conocimiento entre Matemáticas y Física. El objetivo de este artículo es caracterizar las manifestaciones de transferencia o evidencia de conocimiento entre ambas áreas en estudiantes de Bachillerato (16-18 años) cuando resuelven problemas sobre la velocidad media. El enfoque metodológico es cualitativo. Nuestro instrumento de recogida de datos consiste de dos tareas sobre velocidad media en distintos registros de representación, que realizaron un total de 119 estudiantes españoles. Los resultados han permitido identificar cuatro grupos de estudiantes en relación con la evidencia de conocimiento de la tasa de variación media y la velocidad media, puesta de manifiesto en la resolución de las tareas. Un primer grupo de estudiantes que no usa la velocidad media ni la tasa de variación media, un segundo grupo que usa la tasa de variación media, un tercer grupo que evidencia conocimiento de ambas áreas a partir de determinados datos y un cuarto grupo que manifiesta la transferencia de conocimiento entre ambas áreas a partir de cualquier tipo de datos. Estos resultados nos han permitido caracterizar una posible progresión en la transferencia de conocimiento entre ambas áreas en la resolución de tareas sobre velocidad media.

PALABRAS CLAVE:

Transferencia de conocimiento; Matemáticas; Física; velocidad media; Bachillerato.

\section{ABSTRACT}

Results of previous investigations have shown that the transfer of knowledge between Mathematics and Physics can be very complex. The aim of this article is to characterize the manifestation of knowledge transfer between both areas in high school students (16-18 years old) when they solve problems about average speed. The methodological approach selected is qualitative. As a data-collection instrument we used a two-task questionnaire about average speed given in different representation registers. This questionnaire was completed by a total of 119 Spanish students. The resolution of the tasks by the students allowed us to characterize four different groups of students in relation to the evidence of the transfer of knowledge between Mathematics and Physics around the concept of average speed. A first group of students who do not use the average speed or the average rate of variation, a second group who use the average rate of variation, a third group who manifest knowledge transfer between both areas based on certain data and a fourth group that manifest the transfer of knowledge between both areas from any type of data. These results have allowed us to characterize a possible progression in the transfer of knowledge between both areas for the concept of average speed.

\section{KEYWORDS:}

Transfer of knowledge; Mathematics; Physics; Average

Speed; High School students. 


\section{Introducción}

Las leyes físicas son el mejor modelo matemático del que disponemos para describir el comportamiento de nuestro entorno, desde la interacción gravitatoria, las leyes de la termodinámica, etc. Todas estas leyes han sido elaboradas como una aplicación de las Matemáticas para resolver problemas de la vida real (Verschaffel et al., 2002). Parece indudable, entonces, que la Física se ha beneficiado de los avances de las Matemáticas, apoyándose en esta para su propio desarrollo.

Sin embargo, si analizamos la relación entre la Física y las Matemáticas, observamos que el beneficio se da en ambos sentidos. Problemas concretos de Física, como pueden ser aquellos de Cinemática (rama de la Física que estudia el movimiento, sin tener en cuenta las causas que lo produce), han sido cruciales en el desarrollo histórico de las Matemáticas. Sin ir más lejos, el concepto de Derivada estudiado por Newton tuvo su origen en el análisis de la variación de un movimiento (Azcárate, 1990).

Deteniendo la mirada en el sistema educativo español observamos que esta relación aparece truncada. La Física comienza a estudiarse en el segundo curso de la Educación Secundaria Obligatoria (13-14 años), y es entonces cuando aparecen por primera vez en el currículo los conceptos de Cinemática tales como: velocidad media, instantánea y aceleración (Ministerio de Educación, Ciencia y Deporte [MECD], 2015). No obstante, en Matemáticas el concepto de Derivada no se introduce hasta entrado en Primero de Bachillerato (etapa no obligatoria, 16-17 años). Esto supone, tal y como señalan Valera et al. (1983), que la relación de los conceptos de velocidad y aceleración con los de espacio y tiempo sea de muy difícil asimilación por parte de los estudiantes, pese a que su origen se remonte al inicio del estudio del Cálculo, ocasionando que estos estudiantes tengan problemas a la hora de enfrentar enunciados de Mecánica (Azcárate, 1984).

En este sentido, los resultados de diversas investigaciones (McDermott et al., 1987; Planinic et al., 2012) con estudiantes de Educación de Secundaria (15-16 años) concluyen que estos no se dan cuenta de que están trabajando con un mismo elemento matemático cuando resuelven tareas en diferentes contextos, es decir, cuando resuelven tareas de Matemáticas o Física (Planinic et al., 2012). Así, estudiantes que son capaces de resolver una tarea matemática, fallan en la tarea análoga en el contexto físico. Una de las principales fuentes de dificultad de dichos estudiantes, según estos autores, se encuentra en la relación que deben establecer entre los gráficos de contextos físicos, y del mundo real. Sin embargo, investigaciones (Marrongelle, 2001, 2004) centradas en el uso de la Física para resolver problemas de Cálculo a través de un curso integrado de Física y Matemáticas, concluyen que los estudiantes entienden mejor las representaciones gráficas cuando las relacionan con distintos fenómenos físicos, es decir, cuando las contextualizan. En relación con la tasa de variación media, según estas investigaciones, los estudiantes de Física se basan en conceptos físicos para construir conceptualizaciones significativas de la tasa de variación media. Sin embargo, no sucede lo mismo para conceptos como la Derivada o Integral. En esta misma línea, investigaciones realizadas con estudiantes universitarios centradas en el estudio de las respuestas a tareas de aplicación de conceptos matemáticos y del análisis matemático a la Física (Beichner, 1994; Christensen y Thompson, 2012; Quinn, 2013; Thompson et al., 2010) exponen que los estudiantes universitarios cometen errores a la hora de resolver problemas de Cinemática cuando las tareas requieren el cálculo de áreas bajo curvas y pendientes, dificultades que podrían tener su origen en los procesos matemáticos de integración y derivación (Beichner, 1994). Estas investigaciones concluyen que el problema de la transferencia de conocimiento proviene de que los estudiantes carecen de los conceptos previos de Matemáticas necesarios para resolver las tareas desafiantes que se les proponen (Christensen y Thompson, 2012; Thompson et al., 2010). En este sentido, algunas de estas investigaciones concluyen que los estudiantes muestran una menor confianza en realizar una transferencia de conocimiento entre las Matemáticas y la Física y Química cuando trabajan con el concepto matemático de pendiente (Quinn, 2013).

Es decir, la transferencia solo puede producirse cuando se ha construido un esquema coherente y robusto en el dominio inicial del aprendizaje (Rebello et al., 2017). Por otra parte, los resultados de la investigación de Woolnough (2000) muestran que, incluso aquellos estudiantes que normalmente obtienen buenos resultados en ambas disciplinas, fallan al establecer relaciones entre estas, atribuyéndolo a las creencias que tienen los estudiantes con respecto a cada una de las áreas; uno de los estudiantes llega a afirmar que "áreas diferentes hacen las cosas diferentes" (Woolnough, 2000, p. 264).

Como consecuencia de los resultados de todas estas investigaciones, podemos afirmar que el conocimiento matemático no es garantía de éxito en la resolución de tareas físicas, "la componente de interpretación de cantidades matemáticas en el contexto físico (por ejemplo, reconocer la pendiente de un gráfico velocidad-tiempo como la aceleración) falta a veces" (Planinic et al., 2012, p. 1140).

Redish y Kuo (2015) van más allá, concluyendo incluso que, pese a que la falta de éxito con las Matemáticas en los contextos físicos tradicionalmente se atribuye a la falta de habilidades para transferir el conocimiento matemático a las clases de Física, su origen también puede deberse a que "aprender Matemáticas en la clase de Matemáticas y Matemáticas en la clase de Física debe tratarse como si se aprendieran dos lenguajes relacionados pero distintos" (Redish y Kuo, 2015, p. 587) 
Desde la perspectiva de esta problemática se plantea una investigación que pretende caracterizar el desarrollo del esquema de Derivada aplicada a problemas de la Cinemática en estudiantes de Bachillerato (16-18 años). En el transcurso de la misma, se observó distintas manifestaciones en la transferencia de conocimiento que se originaban entre la Física y las Matemáticas en torno a distintos conceptos de la Cinemática. En particular, en este trabajo nos centramos en las asociadas al concepto de tasa de variación media (a partir de ahora, T.V.M.) y velocidad media.

Definimos la T.V.M. en registro algebraico-numérico como el cociente de la variación de una función en un intervalo $[\mathrm{a}, \mathrm{b}]$ entre la variación $b-a$ (1). En el contexto de la Física, definimos la velocidad media vectorialmente como la razón del vector desplazamiento con el intervalo temporal en el que transcurre dicho desplazamiento (Young y Freedman, 2009).

$$
T \cdot V \cdot M_{[a, b]}=\frac{f(b)-f(a)}{b-a}
$$

En el registro de representación gráfico la T.V.M. es la pendiente de la recta secante a la curva en los puntos $(a, f(a)) y(a+h, f(a+h))$. De igual forma, la velocidad media se corresponderá con la pendiente de la recta secante entre dos puntos de la gráfica posicióntiempo.

Por tanto, en términos de transferencia de conocimiento, se puede considerar que la velocidad media es la T.V.M. aplicada al caso particular de que la variable independiente sea el tiempo y la variable dependiente sea la posición del móvil respecto al tiempo. Para el caso particular de esta investigación, trabajaremos con tareas donde el movimiento se produzca en una sola dimensión de forma rectilínea. En estas condiciones, no es necesario el tratamiento vectorial de la velocidad, y la velocidad media podrá definirse como se expresa en (2).

$$
v_{\text {media }}=\frac{x\left(t_{f}\right)-x\left(t_{i}\right)}{t_{f}-t_{i}}
$$

Además, consideraremos que, al tratarse en todas las tareas de movimientos rectilíneos, el signo de la velocidad media nos proporcionará el sentido de la velocidad con respecto a nuestro sistema de referencia.

Este artículo tiene como objetivo caracterizar las manifestaciones de la transferencia de conocimiento entre las Matemáticas y la Física en los estudiantes de Bachillerato (16-18 años) cuando resuelven problemas sobre la velocidad media.

\section{Marco teórico}

La transferencia de conocimiento a menudo se define como la aplicación de lo que uno ha aprendido en una situación a otra situación diferente (Rebello et al., 2017; Reed, 1993; Singley y Anderson, 1989). Una de las concepciones del término transferencia en Educación Matemática descrita por Evans (1999) la considera como la aplicación de la materia académica de Matemáticas fuera de su dominio, en particular en este trabajo la consideramos aplicada a la Cinemática en la Física.

Los modelos tradicionales se centran en aspectos cognitivos (Bassok, 1990; Brown y Kane, 1988; Reed, 1993; Singley y Anderson, 1989). Según Singley y Anderson (1989), el estudiante construye una representación mental abstracta o esquema a través de experiencias en situaciones de aprendizaje y despliega los esquemas en la situación de transferencia. Sin embargo, la mayoría de los estudiantes no puede reconocer similitudes entre el contexto de aprendizaje y el contexto de transferencia y, por lo tanto, no puede resolver con éxito los problemas en el último contexto, a pesar de que pueda hacerlo en el contexto de aprendizaje original. Los investigadores han explicado a menudo la falta de dicha transferencia en términos de la incapacidad de los estudiantes para construir un esquema coherente en el dominio de aprendizaje (Reed, 1993).

Algunas de estas investigaciones (Bransford y Schwartz, 1999; Greeno et al., 1993; Lobato, 1996) han comenzado a considerar la transferencia como las habilidades de los estudiantes para aprender a resolver problemas en el nuevo dominio (Bransford y Schwartz, 1999). Además, estas investigaciones se han centrado en la activación de conocimientos (DiSessa, 1993) o recursos cognitivos (Hammer, 2000) en el nuevo dominio y en la construcción dinámica de similitudes entre el aprendizaje y el contexto de transferencia (Lobato, 2003).

En este sentido, los modelos contemporáneos de transferencia han ido más allá de centrarse únicamente en los aspectos cognitivos de la transferencia, han incluido otros factores mediadores que afectan la transferencia. Una característica común de todas estas perspectivas es que consideran la transferencia como un proceso dinámico activo, y conciben la transferencia como la construcción personal de similitudes entre actividades donde los estudiantes ven las situaciones como similares (Greeno et al., 1993).

Así, desde la perspectiva de Rebello et al. (2005), hay dos tipos de asociaciones que un estudiante puede hacer en un escenario de resolución de problemas. El primer tipo de asociación implica asignar información leída de un problema a un elemento de conocimiento previo del estudiante. Un ejemplo es leer un valor numérico del enunciado del problema y asignarlo a una cantidad física particular. Por ejemplo, si un 
problema indica que un automóvil se está moviendo a 20 metros/segundo, el alumno reconoce que los 20 metros/segundo es la "velocidad" del automóvil, más específicamente que " $v=20 \mathrm{~m} / \mathrm{s}$ ", y puede conectarse a una fórmula ya aprendida o a la derivada de una función, siendo estas parte del esquema interno del alumno para resolver el problema. Este tipo de asociaciones, entre la nueva información obtenida del problema y los elementos de la estructura de conocimiento del estudiante, generalmente están firmemente establecidas por el estudiante y las articula fácilmente. $Y$ también, se produce un segundo tipo de asociación entre un elemento de conocimiento leído del problema y un elemento de la estructura de conocimiento del alumno, que a su vez se basa en su conocimiento previo. Esta asociación suele ser más abstracta y tenue y, a menudo, el alumno puede no ser capaz de articularla claramente. Por ejemplo, un estudiante al que se le muestra una animación de un automóvil en movimiento, sin siquiera saber que la velocidad tiene algo que ver con el problema, comienza a pensar en la velocidad del automóvil como una característica importante del problema. Este alumno está haciendo una asociación implícita entre dos ideas: movimiento (que se muestra en la animación del problema) y velocidad (cuyo conocimiento se considera necesario para describir el movimiento).

Estos dos tipos de asociaciones se pueden considerar vinculadas a dos tipos diferentes de procesos de transferencia. En el primer tipo de transferencia, la transferencia "horizontal", el estudiante lee información proporcionada explícitamente de un escenario problemático y activa su estructura de conocimiento. Esta conexión o asignación entre la información proporcionada y la estructura de conocimiento del estudiante determinará si este puede resolver el problema. Si dicha conexión o asignación no ocurre naturalmente, es decir, si la representación externa del problema no coincide con la estructura de conocimiento del estudiante o la representación interna del problema, este no puede resolver el problema. El enunciado del problema proporciona explícitamente toda la información requerida.

En el segundo tipo de transferencia, la transferencia "vertical", un estudiante reconoce las características de la situación y activa intuitivamente elementos de su conocimiento previo. En este tipo de transferencia, el estudiante generalmente no tiene una estructura de conocimiento preconcebida que se alinee con la información del problema. Más bien, construye un modelo mental in situ a través de sucesivas construcciones y deconstrucciones de asociaciones entre elementos de conocimiento. Por ejemplo, en lugar de que le digan la velocidad inicial y la aceleración del vehículo, al estudiante se le muestra un video o una animación de un vehículo y se le pide que descubra qué distancia pudo haber recorrido el vehículo después de salir del borde del video. En ningún momento se le habla al estudiante de la velocidad o aceleración inicial, ni siquiera de que estas variables sean relevantes para la situación. En este caso el estudiante, fijándose en el plano inclinado, primero debe reconocer que el vehículo estaba acelerando e incluso puede enfrentar el supuesto de que esta aceleración puede no ser uniforme. El estudiante no puede activar una estructura de conocimiento preconcebido claramente identificable o una representación interna que se adecue perfectamente con la situación.

Hay que destacar que un proceso dado puede tener componentes de transferencia "horizontal" y "vertical", y que estos dos procesos no son mutuamente excluyentes. En este trabajo nos centraremos en el proceso de transferencia "horizontal" en relación con la T.V.M. y la velocidad media. Consideraremos que hablamos de transferencia "horizontal" pues presentaremos a los estudiantes situaciones problemáticas con toda la información necesaria para su resolución, esperando que estos activen sus estructuras de conocimiento, tanto aquellas estructuras del dominio de la Física como de las Matemáticas, puesto que ambas son necesarias para resolver la situación. La transferencia de conocimiento se considerará efectuada de forma completa y correcta cuando el estudiante pueda realizar dicha conexión entre la situación problemática y las estructuras en ambos dominios. En este contexto, nuestra pregunta de investigación es la siguiente:

¿Qué transferencia o evidencia de conocimiento entre las Matemáticas y la Física se ponen de manifiesto en los estudiantes de Bachillerato (16-18 años) cuando resuelven problemas sobre la velocidad media?

\section{Metodología}

\subsection{Participantes y contexto}

Los participantes en la investigación fueron 119 estudiantes de la materia de Matemáticas II de Segundo de Bachillerato (17-18 años). Estos estudiantes han sido seleccionados de cuatro centros de Educación Secundaria de la provincia de Sevilla (España). Dichos centros se eligieron por la disponibilidad y facilidad que ofrecieron a la hora de poder realizar este estudio. Como hemos mencionado anteriormente, el concepto de velocidad media se introduce en segundo curso de Educación Secundaria Obligatoria (13-14 años), mientras que es en cuarto curso de la Educación Secundaria Obligatoria (15-16 años) cuando el estudiante aprende el concepto de T.V.M. (MECD, 2015) en la asignatura de Matemáticas orientada a las enseñanzas académicas. $Y$ es en Primero de Bachillerato cuando el concepto de T.V.M. se estudia en Matemáticas y el de velocidad media en Física. Sin embargo, estos contenidos se trabajan en dichas asignaturas -Matemáticas y Física y Química de Primero de Bachillerato- en diferentes momentos del curso (incluso en diferentes trimestres). Por este motivo, se ha considerado que los participantes en nuestra investigación sean estudiantes de un curso 
superior, antes de haber impartido estas materias en Segundo curso (17-18 años).

\subsection{Instrumento de recogida de datos}

El instrumento de recogida de datos seleccionado para este artículo son dos tareas (véase la Figura 1) que forman parte de un cuestionario diseñado para una investigación más extensa. La selección de tareas del cuestionario se ha realizado conforme a tareas utilizadas en investigaciones previas de la Cinemática y del concepto de Derivada, en el ámbito de investigación de Didáctica de las Ciencias Experimentales y Matemáticas, respectivamente. Además, se han adaptado las mismas para la etapa educativa pertinente.

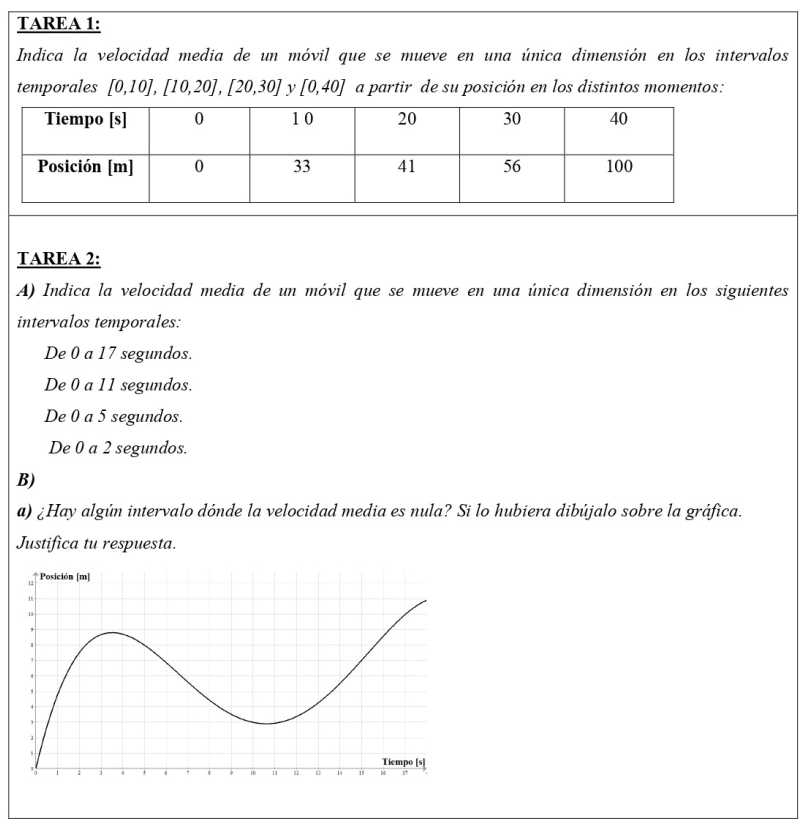

Figura 1. Tareas propuestas a los estudiantes de Bachillerato.

Fuente: Elaboración propia.

La primera tarea dada en registro numérico, como tabla de valores de posición-tiempo, no se corresponde ni al movimiento rectilíneo uniforme ni al movimiento rectilíneo uniformemente acelerado, es decir, no se corresponde con ningún tipo de movimiento estudiado en la instrucción previa por los participantes en esta investigación. Esta tarea se asemeja a la utilizada por Azcárate (1990), no obstante, en su investigación no pregunta acerca de la velocidad media (T.V.M.). Además, en la resolución de esta tarea se espera que el estudiante proceda a calcular la velocidad media de los intervalos temporales proporcionados. Para resolverla, pueden aplicar la fórmula de la T.V.M. desde los datos numéricos. Para ello, los estudiantes deben conectar dos puntos diferentes pero cercanos en un registro algebraico-numérico, es decir, usar dos puntos consecutivos de una tabla que relaciona las dos magnitudes covariantes (posición-tiempo) para calcular la velocidad media.

La segunda tarea, adaptada de investigaciones previas (Azcárate, 1990; Marrongelle, 2004; SánchezMatamoros, 2004), está dada en registro gráfico, y en ella se espera que el estudiante calcule la velocidad media directamente desde la gráfica como la pendiente de la recta secante entre dos puntos de la gráfica, es decir, el estudiante debe relacionar las dos magnitudes covariantes (posición-tiempo) mediante un segmento de línea (debe conectar dos puntos en una curva definida por la gráfica de posición-tiempo para obtener una cuerda, segmento de una línea secante a la curva, que pasa por esos dos puntos), y calcular su pendiente. También puede extraer los datos numéricos a partir de la representación gráfica (en cuyo caso realizará la traslación del registro de representación gráfico al algebraico-numérico) y calcular la velocidad como T.V.M. de la posición con respecto al tiempo. En ambos casos, obtendrá el valor de la velocidad media para cada uno de los intervalos del enunciado. Al igual que en la tarea 1, la gráfica presentada de la posición frente al tiempo muestra que el movimiento que se describe no se corresponde ni al movimiento rectilíneo uniforme ni al movimiento rectilíneo uniformemente acelerado, estudiados en la instrucción previa. Además, en esta tarea deben indicar si hay algún intervalo donde la velocidad media sea nula, esto sucederá cuando el incremento de la posición sea nulo o la pendiente de la recta secante sea cero.

La transferencia o evidencia de conocimiento entre las Matemáticas y la Física puede observarse en la resolución de estas tareas en la medida en que los estudiantes identifican las dos magnitudes covariantes e interpretan el significado físico de lo que les piden, es decir, de la velocidad media. Además de entender su significado físico, los estudiantes deben mostrar que son capaces de trabajar con las tres magnitudes (posición, tiempo y velocidad), haciendo uso de las unidades de medida correspondientes a estas magnitudes. Así mismo, el hecho de que los datos presentados en estas tareas no se corresponden a ninguno de los tipos de movimientos estudiados en su instrucción previa, hace que el estudiante no pueda recurrir a ninguna de las fórmulas aprendidas.

Por tanto, en la resolución de ambas tareas se evidenciará una transferencia de conocimiento "horizontal" por parte del estudiante cuando asocie la velocidad media pedida (contexto físico) con la T.V.M. (contexto matemático) en cada intervalo en la tarea 1, o con la pendiente de la línea secante en la tarea 2. El estudiante leerá la tabla de valores proporcionada en el enunciado (posición-tiempo) en la tarea 1, o los puntos de la gráfica en la tarea 2, y activará su estructura de conocimiento para resolver el problema, dando como resultado del mismo el valor numérico de la velocidad media expresada en " $\mathrm{m} / \mathrm{s}$ " como unidad de magnitud en cada uno de los intervalos temporales de los enunciados de dichas tareas. Además, el hecho de que 
el estudiante sea capaz de responder que la velocidad media será nula (al responder el apartado B de la tarea 2) cuando el incremento de la posición sea nulo, o la pendiente de la recta secante sea cero, también será indicativo de dicha transferencia de conocimiento o evidencia de conocimiento entre Matemáticas y Física, ya que tanto en un caso como en el otro deben usar conocimiento que proviene de las Matemáticas, para considerar que para que la velocidad media sea nula, el numerador del cociente incremental debe ser cero o la recta secante paralela al eje del tiempo. Esto le permitirá concluir, en ambos casos, que la posición del móvil en el instante de tiempo inicial y final de intervalo temporal considerado debe ser la misma.

\subsection{Análisis}

El enfoque metodológico seleccionado para esta investigación es el cualitativo descriptivo. Esta elección se debe a las preguntas de investigación planteadas, y por ende al elemento de recogida de datos utilizado: un cuestionario de preguntas abiertas (Hernández Sampieri et al., 2006). Tal y como se ha visto en el apartado anterior, el cuestionario constará de tareas donde los estudiantes deberán mostrar el proceso de resolución de las mismas y la justificación a dicho proceso.

Para analizar los datos se tomó primero una pequeña muestra a partir de la cual se codificaron las respuestas de los estudiantes a las dos tareas en función a las evidencias, y se crearon varias categorías. Una vez llegado a un acuerdo, se añadieron nuevos datos con el objetivo de revisar el sistema de categorías creado inicialmente y constatar su validez (Strauss y Corbin, 1994). Este proceso de análisis se realizó en dos fases. En la primera fase se analizaba si en la respuesta del estudiante a la tarea se ponía de manifiesto el uso de la T.V.M. o de la velocidad media. En la segunda fase analizamos si los estudiantes de Bachillerato ponían de manifiesto transferencia de conocimiento entre las Matemáticas y la Física o evidenciaban conocimiento de una de ellas o de ambas en la resolución de las tareas. Como resultado de este análisis obtuvimos distintos grupos de estudiantes basados en la manera en la que estos ponían de manifiesto evidencia de conocimiento entre Matemáticas y Física. Así consideramos, por un lado, a los estudiantes que, haciendo un uso correcto de la T.V.M., no evidenciaban conocimiento de Física $y$, por otro, los que haciendo un uso correcto de la T.V.M. además ponían de manifiesto en su resolución diferentes características de conocimiento de ambas. Los resultados de estas fases del análisis están descritos en la siguiente sección.

\section{Resultados}

Esta sección de resultados la hemos estructurado en cuatro apartados. En primer lugar hemos considerado aquellos estudiantes en los que no se evidencia el uso de la T.V.M., en segundo lugar los estudiantes en los que se evidencia el uso de la T.V.M. pero no se evidencia conocimiento de Física, y finalmente aquellos estudiantes en los que se evidencia el uso de la T.V.M. para encontrar la velocidad media y se evidencia además conocimiento de ambas, diferenciando en este último caso dos grupos, los que evidencian conocimiento de Matemáticas y Física en determinadas situaciones y los que evidencian siempre conocimiento de ambas, poniendo de manifiesto la transferencia de conocimiento entre la T.V.M. y la velocidad media (ver Tabla 1). Pasamos a describir cada uno de estos grupos.

Tabla 1

Número de estudiantes asignados a cada grupo

\begin{tabular}{|l|l|}
\hline Grupo & $\begin{array}{l}\text { Número de } \\
\text { estudiantes }\end{array}$ \\
\hline $\begin{array}{l}\text { No uso de la velocidad media ni de la } \\
\text { T.V.M. }\end{array}$ & 46 \\
\hline Uso de la T.V.M. & 8 \\
\hline $\begin{array}{l}\text { Uso de velocidad media y T.V.M. a } \\
\text { partir de datos de posición y tiempo }\end{array}$ & 55 \\
\hline $\begin{array}{l}\text { Uso de velocidad media y T.V.M. a } \\
\text { partir de cualquier tipo de datos }\end{array}$ & 10 \\
\hline Total & 119 \\
\hline
\end{tabular}

Fuente: Elaboración propia.

\subsection{No uso de la velocidad media ni de la tasa de} variación media

En este grupo encontramos 46 estudiantes que no utilizan de forma correcta la velocidad media ni la T.V.M. para resolver las tareas. La mayoría de los estudiantes de este grupo hacen uso del cociente entre posición y tiempo (sin calcular incrementos), un ejemplo de ello lo tenemos en el estudiante E38, como muestra la Figura 2. En la tarea 1, para calcular la velocidad media en el intervalo $[0,10]$ este estudiante coge el valor de la posición del móvil $(33 \mathrm{~m}$ ) en el instante de tiempo correspondiente (10 segundos) para responder que la velocidad en ese intervalo es $3,3 \mathrm{~m} / \mathrm{s}$, actuando de igual forma en los restantes intervalos temporales.

Estos estudiantes identifican parcialmente las magnitudes consideradas en el contexto físico. No distinguen entre posición y distancia recorrida, de ahí que realicen el cociente de la primera de ella entre el tiempo sin atender a incremento. El estudiante mencionado usa una fórmula memorizada en la instrucción previa pero no la recuerda de forma correcta. Ello hace que no pueda asociar el elemento físico de la velocidad media al elemento matemático de la T.V.M. Es decir, recuerda que debe hacer el cociente de la magnitud que tiene unidades de longitud entre la que tiene unidades temporales, pero sin prestar atención a las características de los datos que le da el problema. Observamos que no se produce 
transferencia de conocimiento entre el contexto físico dado y los conocimientos de Matemáticas que tiene el estudiante, debido a que este no interpreta de forma correcta el contexto físico.

Además, se evidencia en el estudiante dificultad en relación con el conocimiento de las Matemáticas, pues al realizar el cociente de la posición inicial (cero metros) entre el tiempo inicial (0 segundos), obtiene el cociente $\mathrm{O} / \mathrm{O}$. Este cociente en Matemáticas se considera una indeterminación, mientras que, en el contexto físico presupone de forma errónea que al estar en la posición 0 metros a los 0 segundos se supone una velocidad $0 \mathrm{~m} / \mathrm{s}$, cuando no es así. Es decir, comenzar a medir el tiempo ( $t=0$ segundos) justo cuando pasa por la posición de referencia ( 0 metros) no tiene por qué indicar que el movimiento tenga velocidad inicial nula. Esto puede considerarse una evidencia de que E38 no asocia el conocimiento de las Matemáticas a la Física, o que en ocasiones las dificultades relacionadas con conocimiento proveniente de las Matemáticas (en nuestro caso, T.V.M. o 0/0) impiden que se produzca la transferencia de conocimiento.

PROCESO DE RESOLUCION (especifica todos los pasos que llevan a la resolución de la tarea)

$$
\begin{aligned}
& v=s / l \quad v_{r}=\frac{0}{0}=0 \mathrm{~m} / \mathrm{s} \\
& \begin{array}{ll}
v_{1}=\frac{33}{10}=3^{\prime 3} \mathrm{~m} / \mathrm{s} \\
v_{2}=\frac{41}{20}=2.05 \mathrm{~m} / \mathrm{s}
\end{array} \\
& \begin{array}{l}
\text { (1) Calculangs bodas } v_{3}=\frac{36}{30}=1^{\prime} 87 \mathrm{~m} / \mathrm{s} \\
\text { las velecidades en conda } \\
\text { intervalo de tie mp. } \\
v_{4}=\frac{100}{40}=2 \mathrm{sm} / \mathrm{s}
\end{array}
\end{aligned}
$$

Figura 2. Respuesta de la tarea 1 del estudiante E38.

$$
\text { Fuente: Datos recogidos. }
$$

\subsection{Uso de la tasa de variación media}

De los 119 estudiantes, 8 utilizan correctamente la fórmula de la T.V.M., pero se evidencia como algo aprendido de la instrucción previa. No ponen de manifiesto evidencia de conocimiento entre la Física y las Matemáticas. Un ejemplo de ello lo tenemos en el estudiante E90 (véase la Figura 3). Este estudiante hace uso correcto de la T.V.M. en los distintos intervalos temporales, pero no indica, en ninguno de ellos, la unidad de medida de las magnitudes consideradas. Además, indica en la justificación de la respuesta que es una fórmula que recuerda de la instrucción previa. En este grupo observamos que el estudiante usa un elemento matemático que ha estudiado previamente, la T.V.M. Sin embargo, a la hora de dar respuesta a la tarea, olvida que la tarea está dada en un contexto físico y responde directamente desde las Matemáticas.

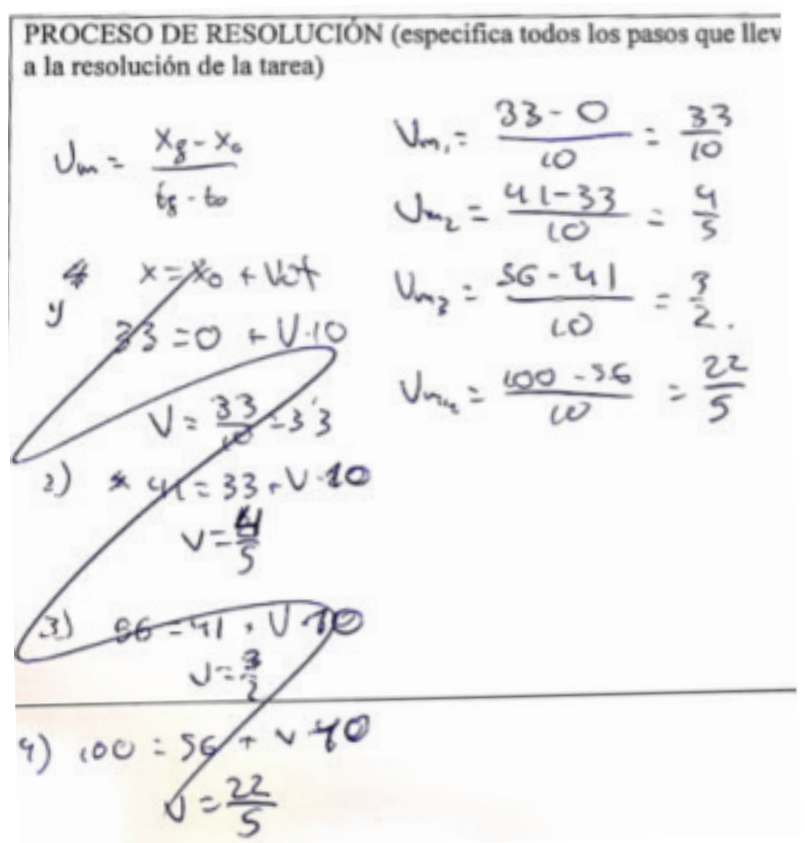

Figura 3. Respuesta de la tarea 1 del estudiante E90.

Fuente: Datos recogidos.

\subsection{Uso de la velocidad media y de la tasa de variación media a partir de datos de posición y tiempo}

En este grupo encontramos 55 estudiantes que utilizan la T.V.M. para calcular la velocidad media de forma correcta, aunque no son capaces de explicar en qué condiciones la velocidad media será nula. Estos estudiantes ponen de manifiesto en la resolución de ambas tareas evidencias de conocimiento entre Matemáticas y Física, al haber asociado la velocidad media pedida con la T.V.M. en cada intervalo en ambas tareas (lo que podría considerarse evidencia de transferencia de conocimiento horizontal), es decir, estos estudiantes han leído la tabla de valores proporcionada en el enunciado (posición-tiempo) en la tarea 1, o los puntos de la gráfica en la tarea 2, y han activado su estructura de conocimiento para resolver el problema, dando como resultado de las mismas el valor numérico de la velocidad media expresada en " $\mathrm{m} / \mathrm{s}$ " como unidad de magnitud en cada uno de los intervalos temporales de los enunciados de dichas tareas. Es decir, desde un contexto físico, estos estudiantes leen la tarea, utilizan el elemento matemático asociado y luego son capaces también de dar la respuesta nuevamente en dicho contexto físico. Además, en algunos de ellos se evidencia también la traslación del registro numérico de los datos al registro gráfico de forma correcta, sin embargo, no son capaces de responder el apartado B de la tarea 2 donde se les pregunta en qué circunstancias la velocidad media es cero, evidenciándose que a partir de la posición y el tiempo dados sí son capaces de evidenciar conocimiento de ambas materias tanto cuando la tarea es dada en registro numérico como en registro gráfico, pero no sucede lo mismo cuando 
a partir de un valor dado de la velocidad media deben deducir qué sucede con la posición del móvil respecto al tiempo (no hay evidencia de transferencia de conocimiento horizontal), un ejemplo sería el estudiante E5 (véase Figura 4).

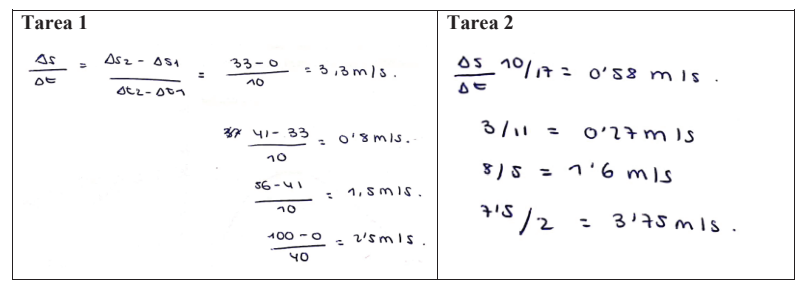

Figura 4. Respuestas de las tareas 1 y 2 del estudiante E5. Fuente: Datos recogidos.

Entre estos estudiantes, hay un grupo (15 de estos 55 estudiantes) que, además de las velocidades medias en cada intervalo, calculan la media o promedio de todas las velocidades medias obtenidas en los distintos intervalos temporales. Un ejemplo de ello lo tenemos en la respuesta del estudiante E68 a la tarea 1 (Figura 5), que además escribe en la justificación de la respuesta: "la velocidad media es la suma de las velocidades de los intervalos partida por el número de estos". Esto no se les pedía en el enunciado de la tarea, por lo que puede considerarse una dificultad añadida en relación a la transferencia de conocimiento entre Matemáticas y Física, puesto que estos estudiantes parecen estar aplicando la media aritmética aprendida en Matemáticas cuando realizan este último cálculo del promedio de las velocidades. Esto se puede considerar una evidencia de que este grupo de estudiantes no conoce las condiciones bajo las que el contenido matemático que han usado (la media aritmética) no es aplicable en Física.

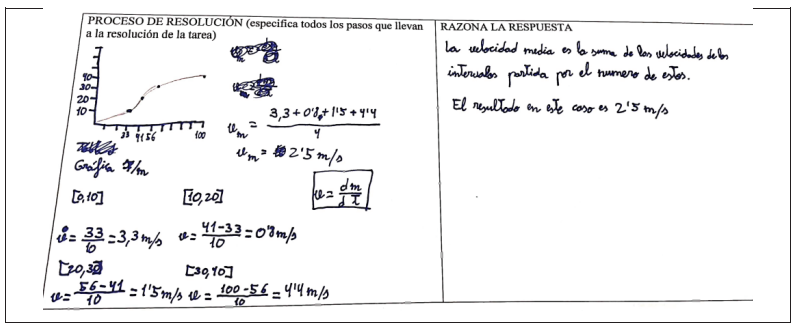

Figura 5. Respuesta de la tarea 1 del estudiante E68 Fuente: Datos recogidos.

\subsection{Uso de la velocidad media y de la tasa de variación media: transferencia de conocimiento}

Finalmente, en este grupo encontramos a 10 estudiantes que utilizan la T.V.M. para dar respuesta a las tareas sobre la velocidad media, y además ponen de manifiesto en su respuesta la transferencia de conocimiento entre la Física y las Matemáticas, es decir, se evidencia conocimiento en todas las situaciones planteadas (lo que podría considerarse evidencia de transferencia de conocimiento horizontal). Estos estudiantes utilizan correctamente la T.V.M. para hallar la velocidad media tanto a partir del registro numérico como del registro gráfico, y utilizan en todo momento las unidades de medida correspondientes a las magnitudes consideradas en la situación planteada para dar una respuesta correcta. Un ejemplo de esto lo encontramos en el estudiante E100, este estudiante en la tarea 1, dada en registro numérico, responde de forma correcta justificando su resolución a partir de la expresión de la velocidad media (incremento de la posición dividido por el incremento del tiempo) expresando el resultado en $\mathrm{m} / \mathrm{s}$ (véase Figura 6).

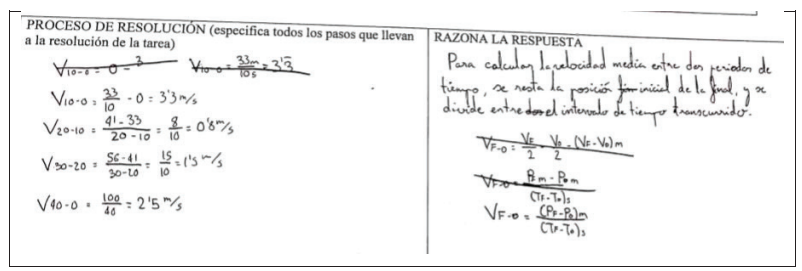

Figura 6. Respuesta de la tarea 1 del estudiante E100.

Fuente: Datos recogidos.

Además, en la tarea 2 (véase Figura 6), dada en registro gráfico, vuelve a poner de manifiesto el uso correcto de la T.V.M. aplicado a la velocidad media. Por tanto, en la resolución de ambas tareas se evidencia por parte del estudiante E100 conocimiento de Matemáticas y Física, lo que podría estar poniendo de manifiesto una transferencia de conocimiento horizontal. El estudiante E100 ha asociado la velocidad media pedida con la T.V.M. en cada intervalo en ambas tareas, es decir, ha leído la tabla de valores proporcionada en el enunciado (posición-tiempo) en la tarea 1, o los puntos de la gráfica en la tarea 2 , y ha activado su estructura de conocimiento para resolver el problema, dando como resultado de las mismas el valor numérico de la velocidad media expresada en " $\mathrm{m} / \mathrm{s}$ " como unidad de magnitud en cada uno de los intervalos temporales de los enunciados de dichas tareas.

Así mismo, responde correctamente al apartado B de la tarea 2. Es decir, indica correctamente que la velocidad media se anula en el intervalo [6, 15], justificando que "para que la velocidad media sea nula el móvil debe estar en la misma posición en varios periodos [refiriéndose a los instantes de tiempo] en este caso como en el séptimo y decimoquinto segundos el móvil está a siete metros, se anula la velocidad media en ese periodo" (véase la Figura 7). Esta respuesta de E100 es una evidencia de conocimiento de Matemáticas y Física (podría ser una manifestación de transferencia de conocimiento horizontal entre ambas materias), ya que ha considerado que para que la velocidad media 
sea nula, la posición del móvil en el instante de tiempo inicial y final del intervalo temporal que se considere debe ser la misma, es decir, el numerador del cociente incremental debe ser nulo (al tratarse del numerador de una fracción, conocimiento de Matemáticas que transfiere a Física). De esta manera, se ha puesto de manifiesto que ha activado su estructura de conocimiento para resolver el problema de forma correcta.

En la resolución de ambas tareas se ha evidenciado, por parte del estudiante, conocimiento de Matemáticas y Física con relación a la T.V.M. y a la velocidad media a partir de cualquier tipo de datos. Es decir, se ha puesto de manifiesto transferencia de conocimiento entre ambas materias por parte del estudiante, de lo que se deduce que este tiene construido un esquema coherente y robusto de la T.V.M.

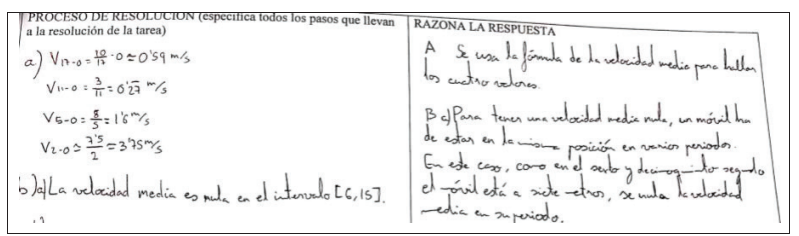

Figura 7. Respuesta de la tarea 2 del estudiante E100. Fuente: Datos recogidos.

\section{Discusión y conclusiones}

El propósito de este artículo es caracterizar las manifestaciones de la transferencia de conocimiento entre la Física y las Matemáticas en la respuesta de los estudiantes de Bachillerato (16-18 años) a problemas sobre la velocidad media. Los resultados del análisis nos han permitido observar cómo hay un primer grupo de estudiantes que recuerda la fórmula de la velocidad media aprendida en la instrucción previa con errores, y un segundo grupo que recuerda la fórmula de la T.V.M. aprendida de la instrucción previa de forma correcta pero sin manifestar transferencia de conocimiento. Estos dos grupos podemos considerar que ven ambas materias, Matemáticas y Física, de forma separada (Woolnough, 2000). En este mismo sentido, una de las conclusiones de la investigación de Planinic et al. (2012) es que los estudiantes no son capaces de asociar los elementos matemáticos con las definiciones de los conceptos físicos, y ello les impide la transferencia de conocimiento entre ambas materias. También, la investigación de Marrongelle (2004) corrobora este resultado, pues, centrándose en el análisis de la transferencia de conocimiento de la T.V.M. en el registro de representación gráfico, observó cómo uno de los estudiantes resuelve el problema de forma rigurosa desde el punto de vista de las Matemáticas, pero no fue capaz de contextualizar su respuesta en el dominio de la Física.

Además, como resultado de este trabajo, hemos obtenido un tercer grupo de estudiantes que pone de manifiesto en la resolución de las tareas evidencias de conocimiento de Matemáticas y Física al haber asociado la velocidad media pedida con la T.V.M. en cada intervalo. Estos estudiantes han leído la tabla de valores proporcionada en el enunciado (posicióntiempo), o los puntos de la gráfica, y han activado su estructura de conocimiento para resolver el problema (Rebello et al., 2005), dando como resultado el valor numérico de la velocidad media expresada en "m/s" como unidad de magnitud en cada uno de los intervalos temporales de los enunciados de dichas tareas. En estos estudiantes se pone de manifiesto evidencia de conocimiento de ambas materias cuando deben calcular la velocidad media a partir de datos relativos a la posición del móvil y el tiempo tanto cuando estos datos vienen dados en registro numérico como en registro gráfico, sin embargo, no sucede lo mismo cuando a partir de un valor dado de la velocidad media deben deducir qué sucede con la posición del móvil respecto al tiempo. Y, por último, obtuvimos un cuarto grupo de estudiantes en los que se evidencia la transferencia de conocimiento entre Matemáticas y Física a partir de cualquier dato, ya que tanto cuando deben calcular la velocidad media a partir de datos relativos a la posición del móvil y el tiempo, como cuando a partir de un valor dado de la velocidad media deben deducir qué sucede con la posición del móvil respecto al tiempo, han activado su estructura de conocimiento para resolver el problema.

Desde estos resultados, se puede inferir en los estudiantes de Bachillerato una progresión en la transferencia de conocimiento entra la Física y las Matemáticas, que comienza en un primer momento no usando la velocidad media ni la T.V.M. de forma correcta; a continuación, en un segundo momento, usando la T.V.M. de forma correcta pero no asociándola a la velocidad media; en un tercer momento, asociando la velocidad media a la T.V.M. a partir de datos de posición y tiempo para calcular la velocidad media, y por último, en un cuarto momento, asociando la velocidad media a la T.V.M. tanto a partir de datos de posición y tiempo para calcular velocidad media, como a partir de datos de la velocidad media para calcular la posición respecto al tiempo. La caracterización de esta transferencia de conocimiento viene recogida en la Figura 8:

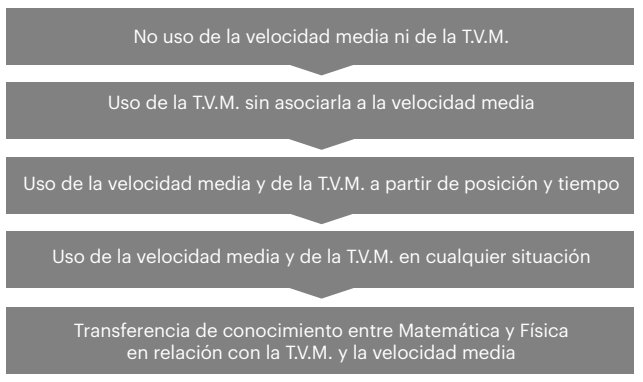

Figura 8. Transferencia de conocimiento entre Matemáticas y Física de la T.V.M. y la velocidad media. Fuente: Elaboración propia. 
Una de las dificultades en la transferencia de conocimiento que se ha puesto de manifiesto en la resolución de las tareas por parte de los estudiantes es la reiteración del cálculo de la velocidad media incluyendo el cálculo de la media aritmética de las velocidades. Aquellos estudiantes que, tras calcular la T.V.M. en cada intervalo, hacen la media o promedio de dichos valores, pueden estar manifestando una confusión entre el término "media" en ambos ámbitos. La media o promedio en Matemáticas se define como la suma de todos los valores obtenidos entre el número de valores (3).

$\bar{v}=\frac{v_{\text {media } 1+} v_{\text {media } 2+} v_{\text {media } 3}+\cdots}{n}=\frac{\sum v_{\text {media }}}{n}$

Este tipo de dificultades en la transferencia de conocimiento entre Matemáticas y Física, que se pone de manifiesto en los estudiantes de Bachillerato, está en coherencia con el resultado de investigaciones previas y puede tener su origen en los procesos matemáticos implicados (Christensen y Thompson, 2012; Thompson et al., 2010), o en la propia Física, debido a que los estudiantes no observan similitudes entre los problemas en Matemáticas y Física (Planinic et al., 2012). Atendiendo a lo que exponen Redish y Kuo (2015), la dificultad puede venir asociada a que los estudiantes están acostumbrados a trabajar en la clase de Matemáticas y Física con lenguajes distintos, imposibilitando esta transferencia de conocimiento.

Todos los estudiantes participantes en el estudio que respondieron la tarea 2 lo hicieron trasladando el enunciado de la tarea, dado en registro gráfico, al registro numérico, ningún estudiante hizo uso de la velocidad media como pendiente de la recta secante entre dos puntos de la curva. Este resultado está en coherencia con resultados de investigaciones anteriores (Planinic et al., 2012) y nos indica que hay un predominio del registro algebraico-numérico sobre el registro gráfico en los estudiantes de Bachillerato. Como expresa Quinn (2013), los estudiantes tienden a evitar el concepto de pendiente en tareas que requieren la transferencia de conocimiento.

Este trabajo nos ha permitido caracterizar la transferencia de conocimiento entre Matemáticas y Física del concepto de velocidad media en relación con la T.V.M. en estudiantes de Bachillerato. Así mismo, al haber preguntado por distintas velocidades medias, ha puesto de relieve la dificultad vinculada al promedio de las velocidades medias, hecho que en investigaciones previas (Marrongelle, 2004) no se había puesto de manifiesto.

\section{Agradecimientos}

El presente trabajo ha recibido el apoyo del Proyecto del Plan Nacional: EDU2017-87411-R del "Ministerio de Economía y Competitividad, Gobierno de España". 


\section{Referencias}

Azcárate, C. (1984). La nueva ciencia del movimiento de Galileo: una génesis difícil. Enseñanza de las Ciencias, 2(3), 203-208.

Azcárate, C. (1990). La velocidad: introducción al concepto de derivada. Universitat Autònoma de Barcelona.

Bassok, M. (1990). Transfer of domain-specific problem-solving procedures. Journal of Experimental Psychology: Learning, 16(3), 522-533. https://doi. org/10.1037/0278-7393.16.3.522

Beichner, R. J. (1994). Testing student interpretation of kinematics graphs. American Journal of Physics, 62(8), 750-762. https://doi.org/10.1119/1.17449

Bransford, J. D., y Schwartz, D. (1999). Rethinking transfer: A simple proposal with multiple implications. Review of Research in Education, 24, 61-100. https://doi. org/10.3102/0091732X024001061

Brown, A. L., y Kane, M. J. (1988). Preschool children can learn to transfer: Learning to learn and learning from example. Cognitive Psychology, 20(4), 493-523. https://doi.org/10.1016/0010-0285(88)90014-X

Christensen, W. M., y Thompson, J. R. (2012). Investigating graphical representations of slope and derivative without a physics context. Physical Review Special Topics - Physics Education Research, 8(2), 1-5. https://doi.org/10.1103/PhysRevSTPER.8.023101

DiSessa, A. (1993). Towards an epistemology of physics. Cognition and Instruction, 10(2-3), 105-225. https://doi.org/10.1080/07370008.1985.9649008

Evans, J. (1999). Building Bridges: Reflections on the Problem of. Educational Studies in Mathematics, 39, 2344. https://doi.org/10.1023/A:1003755611058

Greeno, J. G., Moore, J. L., y Smith, D. R. (1993). Transfer of situated learning. En D. K. Detterman, y R. J. Sternberg (Eds.), Transfer on trial: Intelligence, cognition and instruction (pp. 99-167). Ablex.

Hammer, D. (2000). Student Resources for Learning Introductory Physics. American Journal of Physics - Physics Education Research Supplement, 68(7), S52-S59. https://doi.org/10.1119/1.19520

Hernández Sampieri, R., Fernández Collado, C., y Baptista Lucio, P. (2006). Metodología de la investigación (4.a ed.). McGraw-Hill Interamericana.

Lobato, J. (1996). Transfer reconceived: How "sameness" is produced inmathematical activity. (Tesis doctoral, University of California). Dissertation Abstracts International, AAT 9723086.
Lobato, J. (2003). How Design Experiments Can Inform a Rethinking of Transfer and Vice Versa. Educational Researcher, 32(1), 17-20. http://www.jstor. org/stable/3699930

Marrongelle, K. A. (2001). Physics experiences and calculus: How students use physics to construct meaningful conceptualizations of calculus concepts in an interdisciplinary calculus / physics course. University of New Hampshire.

Marrongelle, K. A. (2004). How Students Use Physics to Reason About Calculus Tasks. School Science and Mathematics, 104(6), 258-272. https://doi. org/10.1111/j.1949-8594.2004.tb17997.x

McDermott, L. C., Rosenquist, M. L., y Van Zee, E. H. (1987). Student difficulties in connecting graphs and physics: Examples from kinematics. American Journal of Physics, 55(6), 503-513. https://doi.org/10.1119/1.15104

Ministerio de Educación, Ciencia y Deporte [MECD] (2015). Real Decreto 1105/2014. Boletín Oficial del Estado, Sec. I (Num. 3), 169-546.

Planinic, M., Milin-Sipus, Z., Katic, H., Susac, A., elvanjek, L. (2012). Comparison of student understanding of line graph slope in physics and mathematics. International Journal of Science and Mathematics Education, 10(6), 1393-1414. https://doi.org/10.1007/s10763-012-9344-1

Quinn, R. (2013). Students' Confidence in the Ability to Transfer Basic Math Skills in Introductory Physics \& Chemistry Courses at a Community College. Dissertations, 438. https://aquila.usm.edu/ dissertations/438

Rebello, N. S., Cui, L., Bennett, A. G., Zollman, D. A., y Ozimek, D. J. (2017). Transfer of Learning in Problem Solving in the Context of Mathematics and Physics. En D. H. Jonassen (Ed.), Learning to Solve Complex Scientific Problems (pp. 223-246). Routledge. https:// doi.org/10.4324/9781315091938-10

Rebello, N. S., Zollman, D. A., Allbaugh, A. R., Engelhardt, P. V., Gray, K. E., Hrepic, Z., Itza-Ortiz, S. F. (2005). Dynamic Transfer: A Perspective from Physics Education Research. En J. P. Mestre (Ed.), Transfer of Learning from a Modern Multidisciplinary Perspective (pp. 217-250). Information Age Publishing Inc.

Redish, E. F., y Kuo, E. (2015). Language of Physics, Language of Math: Disciplinary Culture and Dynamic Epistemology. Science and Education, 24(5-6), 561590. https://doi.org/10.1007/s11191-015-9749-7

Reed, S. K. (1993). A schema-based theory of transfer. En D. K. Detterman, y R. J. Sternberg (Eds.), Transfer on trial: Intelligence, Cognition and Instruction (pp. 39-67). Ablex. 
Sánchez-Matamoros, G. (2004). Análisis de la comprensión en los alumnos de bachillerato y primer año de universidad sobre la noción matemática de derivada (Desarrollo del concepto). (Tesis doctoral, Universidad de Sevilla). Depósito de investigación idUS. https:// idus.us.es/xmlui/handle/11441/73311\#.Xg4kbuasO3I. mendeleyç

Singley, K., y Anderson, J. R. (1989). The Transfer of Cognitive Skill. Harvard University Press.

Strauss, A., y Corbin, J. (1994). Grounded theory methodology: An overview. En N. K. Denzin, y Y. S. Lincoln (Eds.), Handbook of qualitative research (pp. 273-285). Sage Publications, Inc.

Thompson, J., Christensen, W., y Mountcastle, D. (2010). Investigating Student Understanding of Physics Concepts and the Underlying Calculus Concepts in Thermodynamics. Bulletin of the American Physical Society, 55. http://meetings.aps.org/link/BAPS.2010. MAR.H42.3

Valera, M., López Fernández, C., García García, S., Gil Ibáñez, J., Frutos, J., Iniesta, M. A., y Marset, P. (1983). Intuición e historia de las Ciencias en la Enseñanza. Enseñanza de las Ciencias, 1(3), 205-217.

Verschaffel, L., Greer, B., y De Corte, E. (2002). Everyday Knowledge and Mathematical Modeling of School Word Problems. Symbolizing, Modeling and Tool Use in Mathematics Education, (1993), 257-276. https:// doi.org/10.1007/978-94-017-3194-2_16

Woolnough, J. (2000). How do students learn to apply their mathematical knowledge to interpret graphs in physics? Research in Science Education, 30(3), 259-267. https://doi.org/10.1007/BF02461633

Young, H. D., y Freedman, R. A. (2009). Física universitaria (12.a ed., vol. I). Pearson Educación. 